\title{
Compressive-sensing-based Human Action Recognition
}

\author{
Jun Jiang \\ School of Computer Science \\ Southwest Petroleum University \\ Chengdu, China \\ toddjiang@163.com
}

\begin{abstract}
A new compressive sensing based dimensionality reduction method is proposed for human action recognition, in which a novel hybrid random matrix (HRM) is constructed and is proved to satisfy the restricted isometry property. It projects the high-dimensional features into a low-dimensional space via the HRM. Then the low-dimensional features are used for classification. Experimental results demonstrate that the proposed method is effective and efficient in human action recognition, and is on par with or better than the state-of-the-art methods.
\end{abstract}

Keywords-compressive sensing; hybrid fusion matrix; action recognition

\section{INTRODUCTION}

In recent years, human action recognition has been extensively studied in computer vision community in that it finds applications in various areas including intelligent video surveillance, human-computer interaction, and smart robotics. Many action recognition methods use complex highdimensional action features which might lead to unacceptable computational time or storage. To alleviate this problem, dimensionality reduction is adopted to produce lowdimensional features. Various types of dimensionality reduction techniques, such as principal component analysis (PCA), linear discriminant analysis (LDA), multidimensional scaling (MDS), isometric feature map (ISOMAP), and local linear embedding (LLE), have been proposed in the last several decades [1]. However, most of these methods need a datadependent training process and hence result in limited generalization. In addition, they require high computational complexity as they involve time-consuming operations. Recently, compressive sensing (CS) has provided a new way of

\section{Gao Li}

State Key Laboratory of Oil and Gas Reservoir Geology and Development

Southwest Petroleum University

Chengdu, China

lgmichael@263.net

compressing signals [2, 3], and has been applied to image processing [4].

In this paper, we propose a compressive sensing with hybrid random matrix (CSHRM) method for recognizing human actions, where a novel measurement matrix is constructed and is shown to satisfy the restricted isometry property (RIP). As shown in Fig. 1, our method consists of three major components, i.e., feature extraction, dimensionality reduction, and classification. Experimental results demonstrate that the proposed method is effective and efficient to reduce the dimensions of the features, and performs on par with or better than the representative state-of-the-art action recognition methods.

\section{PRoposed METHOD}

\section{A. Background Theory}

Given a vector $\mathrm{x}$ in $\mathrm{R}^{N}$ and a finite-dimensional operator $\Phi$, namely a $M \times N$ measurement matrix in CS, we can obtain a vector $\mathrm{y}$ in $\mathrm{R}^{M}(M<<N)$, such that

$$
\mathbf{y}=\Phi \mathbf{x} .
$$

Furthermore, if $\Phi$ satisfies the RIP, and $\mathbf{x}$ is K-sparse, then $\mathrm{x}$ can be exactly reconstructed from $\mathbf{y}$ with overwhelming probability. $\Phi$ is said to satisfy the RIP if there exists a constant $\delta \in(0,1)$ such that

$$
(1-\delta)\|\mathbf{x}\|_{2}^{2} \leq\|\Phi \mathbf{x}\|_{2}^{2} \leq(1+\delta)\|\mathbf{x}\|_{2}^{2}
$$

holds for all K-sparse vectors in $R^{N}$ [2]. The RIP implies that the metric structure is almost invariant in much lower

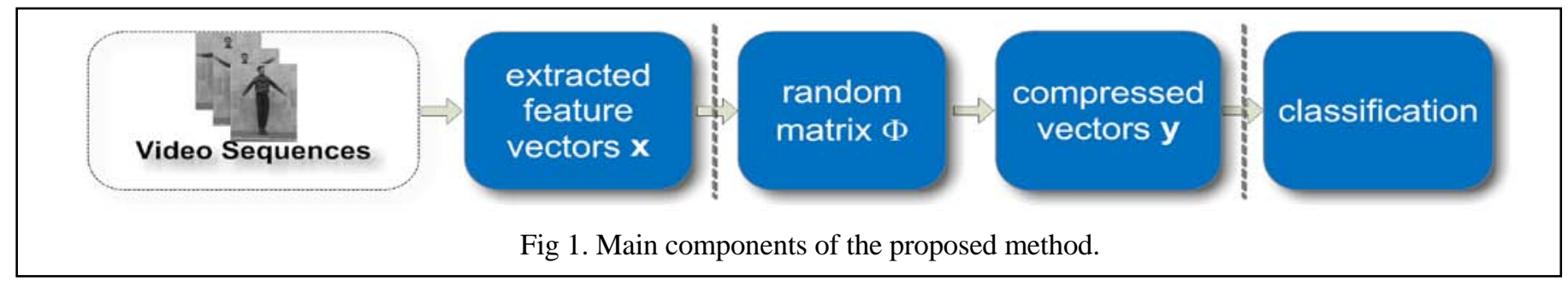


dimensional space, and thus the input data can have a compact representation without losing information.

\section{B. Hybrid random matrix}

Two widely used measurement matrices, i.e., random Gaussian matrix (RGM) and random Bernoulli matrix (RBM), are presented in [5]. Based on these two matrices, we construct a novel measurement matrix called hybrid random matrix (HRM). The HRM, denoted as $\Phi_{H}$, is defined as

$$
\Phi_{H}=(1-\lambda) \Phi_{G}+\lambda \Phi_{B},
$$

where $\lambda \in[0,1], \Phi_{G}$ denotes the RGM, $\Phi_{B}$ denotes the RBM, and $\Phi_{H}, \Phi_{G}, \Phi_{B} \in R^{M \times N}$. Note that the RGM and RBM are two special cases of the HRM when $\lambda=0,1$, respectively.

Proposition 1. Given $\delta \in(0,1)$, there exist positive constants $C_{1}, C_{2}$ depending on $\delta$ such that $\Phi_{H}$ satisfies the RIP with probability $\geq 1-2 e^{-c_{2} M}$ if $M \geq c_{1} K \log (N / K)$.

Proof. We first show

$$
\operatorname{E\Phi }\left(\Phi\left\|_{H} \mathbf{x}\right\|_{2}^{2}\right)=\|\mathbf{x}\|_{2}^{2} .
$$

Without loss of generality, we can assume $\left\|\Phi_{G} \mathbf{x}\right\|_{2} \leq\left\|\Phi_{G} \mathbf{x}\right\|_{2}$, then

$$
\begin{aligned}
& \left.\mathrm{E} \Phi\left\|E_{H} \mathbf{x}\right\|_{2}^{2}\right)=\left(\left\|(1-\lambda) \Phi_{G} \mathbf{x}+\lambda \Phi_{B} \mathbf{x}\right\|_{2}^{2}\right) \leq \\
& \mathrm{E}\left(\left(\left\|(1-\lambda) \Phi_{G} \mathbf{x}\right\|_{2}+\left\|\lambda \Phi_{B} \mathbf{x}\right\|_{2}\right)^{2}\right) \leq\|\mathbf{x}\|_{2}^{2},
\end{aligned}
$$

and we can also get

$$
\begin{aligned}
& \mathrm{E}\left(\Phi\left\|E_{H} \mathbf{x}\right\|_{2}^{2}\right)=\left(\left\|(1-\lambda) \Phi_{G} \mathbf{x}+\lambda \Phi_{B} \mathbf{x}\right\|_{2}^{2}\right) \geq \\
& \mathrm{E}\left(\left(\left\|(1-\lambda) \Phi_{G} \mathbf{x}\right\|_{2}-\left\|\lambda \Phi_{B} \mathbf{x}\right\|_{2}\right)^{2}\right) \geq\|\mathbf{x}\|_{2}^{2},
\end{aligned}
$$

because $\mathrm{E}\left(\left\|\Phi_{G} \mathbf{x}\right\|_{2}^{2}\right)=\|\mathbf{x}\|_{2}^{2}, \mathrm{E}\left(\left\|\Phi_{B} \mathbf{x}\right\|_{2}^{2}\right)=\|\mathbf{x}\|_{2}^{2}$ according to [5].

Combining (5) and (6), we obtain $\mathrm{E}\left(\left\|\Phi_{H} \mathbf{x}\right\|_{2}^{2}\right)=\|\mathbf{x}\|_{2}^{2}$.

Since $\Phi_{B}$ obeys the concentration inequality [6], we have

$$
\begin{aligned}
& \operatorname{Pr}\left(\left|\left\|\Phi_{H} \mathbf{x}\right\|_{2}^{2}-\|\mathbf{x}\|_{2}^{2}\right| \geq \varepsilon\|\mathbf{x}\|_{2}^{2}\right)= \\
& \operatorname{Pr}\left(\left\|\left|(1-\lambda) \Phi_{G} \mathbf{x}+\lambda \Phi_{B} \mathbf{x}\left\|_{2}^{2}-\right\| \mathbf{x}\left\|_{2}^{2} \mid \geq \varepsilon\right\| \mathbf{x} \|_{2}^{2}\right) \leq\right.\right. \\
& \operatorname{Pr}\left(\left|\left(\left\|\Phi_{B} \mathbf{x}\right\|_{2}^{2}-\|\mathbf{x}\|_{2}^{2}\right)\right| \geq \varepsilon\|\mathbf{x}\|_{2}^{2}\right) \leq e^{-d M},
\end{aligned}
$$

where $d$ is a positive constant and depends only on $\delta$.

Hence, by theorem 5.2 in [6], we can derive the proposition.

\section{Action recognition}

First, we use the algorithm presented in [7] to form raw features. Then each original high-dimensional feature $\mathbf{x}$ is transformed to the low-dimensional feature $\mathbf{y}$ by

$$
\mathbf{y}=\Phi_{H} \mathbf{x}
$$

In the classification step, $\mathbf{y}$ is fed into the Support Vector Machine (SVM) for training.

\section{EXPERIMENTAL RESULTS}

We use two benchmark datasets, i.e. WEIZMANN and $\mathrm{KTH}$ [7], to evaluate our method, and conduct the experiments using the leave-one-out cross-validation (LOOCV) strategy. All methods are implemented in Matlab 2011 on a PC with a single Intel Core2 $2.3 \mathrm{GHz}$ processor and $2 \mathrm{~GB}$ memory.

We compare our CSHRM with PCA, MDS, ISOMAP, LLE, CSRGM (compressive sensing with RGM), and CSRBM (compressive sensing with RBM). In the feature extraction phase, we use the same settings as in [7] and thus obtain the 2500-dimensional features. The $\lambda$ of $\Phi_{H}$ is emprically set to be 0.6. The numbers of neighbors for ISOMAP and LLE are set to be 5 and 12, respectively. Finally, in the classification phase, the radial basis function kernel is chosen for the SVM. For fairness, the optimal parameters of all algorithms are selected to achieve the best performance, and the results are obtained by averaging over 20 independent realizations.

Fig.2 shows the accuracy comparisons of all seven dimensionality reduction methods for different dimensions. We can see that the CSHRM outperforms the other algorithms in dimensions ranging from 40 to 400 on both datasets. The best accuracies achieved by the CSHRM are $99.0 \%$ on the WEIZMANN and $97.1 \%$ on the KTH with dimension 400 . Table 1 presents the computational time costs of various algorithms in dimension 400 . Since the feature extraction is the same for all algorithms, we compare merely the dimensionality reduction and classification (training/testing) costs of them. It can be seen that the CSHRM costs the second least, which benefits from the simple and training-free dimension-reduced operation. Moreover, we compare our action recognition approach with the state-of-the-art methods [7] in Table 2. Clearly, our approach is superior to all the other methods on the $\mathrm{KTH}$, and is the second best on the WEIZMANN.

TABLE I. . Computational time costs of seven dimensionality reduction methods on two datasets with dimension 400 .

\begin{tabular}{|c|c|c|}
\hline Method & \multicolumn{2}{|c|}{ Time cost (seconds) } \\
\hline & WEIZMANN & KTH \\
\hline PCA & 1.5 & 15.6 \\
\hline MDS & 4.4 & 12.9 \\
\hline ISOMAP & 11.4 & 24.7 \\
\hline LLE & 10.9 & 33.2 \\
\hline CSBRM & 0.4 & 9.5 \\
\hline CSGRM & 0.9 & 17.1 \\
\hline CSHRM & $\mathbf{0 . 5}$ & $\mathbf{9 . 8}$ \\
\hline
\end{tabular}




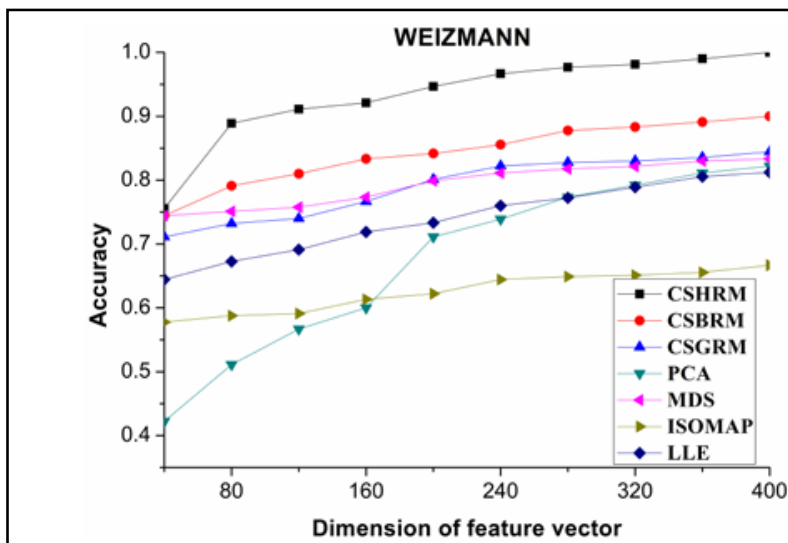

(a)

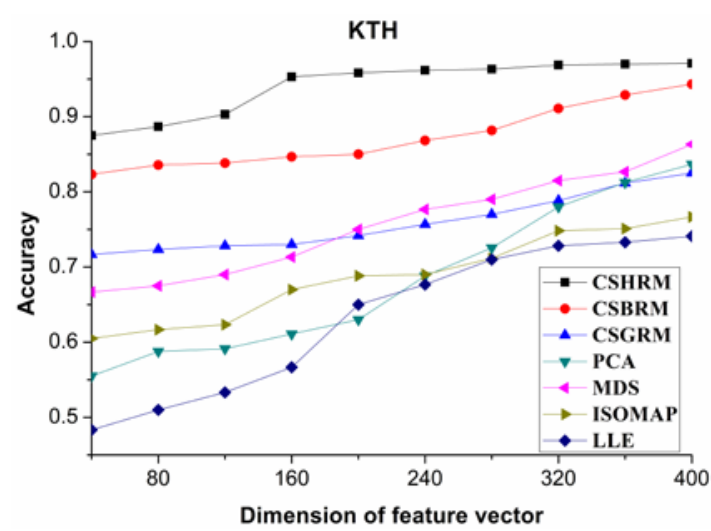

(b)

Fig 2. Recognition accuracy of seven dimensionality reduction methods for different dimensions on two datasets.(a)WEIZMANN (b) KTH.

TABLE II. . Comparison of recognition accuracy with the state-of-theart methods [7]

\begin{tabular}{|c|c|c|}
\hline Method & \multicolumn{2}{|c|}{ Accuracy (\%) } \\
\hline & WEIZMANN & KTH \\
\hline Bregonzio et al. & 96.66 & 94.33 \\
\hline Sun et al. & 97.80 & 94.00 \\
\hline Ikizler et al. & - & 94.00 \\
\hline Lin et al. & - & 93.43 \\
\hline Wang et al. & 100 & 92.51 \\
\hline Liu et al. & - & 92.30 \\
\hline Kläser et al. & 84.30 & 91.40 \\
\hline Niebles et al. & 90.00 & 83.30 \\
\hline Dollar et al. & 85.20 & 81.17 \\
\hline Liu et al. & - & 94.16 \\
\hline Zhao et al. & - & 91.17 \\
\hline Savarese et al. & - & 86.83 \\
\hline CSHRM & $\mathbf{9 9 . 2 0}$ & $\mathbf{9 5 . 1 0}$ \\
\hline
\end{tabular}

\section{CONCLUSION}

In this paper, we have presented the CSHRM for human action recognition. Specifically, a novel HRM is constructed and is shown to obey the RIP. Experimental results demonstrate that our CSHRM outperforms the conventional dimensionality reduction algorithms in terms of efficiency and effectiveness. Furthermore, our action recognition method can give competitively good or even better performance when compared with the state-of-the-art methods.

\section{REFERENCES}

[1] C. Burges, Dimension Reduction: A Guided Tour, Foundations and Trends in Machine Learning, vol.2, no.4, pp. 275-365, 2010.

[2] E. Cande`s and M. Wakin,, An Introduction To Compressive Sampling, IEEE Signal Process. Mag., vol.25, no.2, pp. 21-30, 2008.

[3] D. Donoho, Compressed sensing, IEEE Trans. Inf. Theory, vol.52, no.2, pp. 1289-1306, 2006.

[4] A. Wang, L. Liu, B. Zeng and H. Bai, Progressive image coding based on an adaptive block compressed sensing, IEICE Electron. Express, vol.8, no.8, pp. 575-581, 2011.

[5] D. Achlioptas, Database-friendly random projections: JohnsonLindenstrauss with binary coins, J. Comput. Syst. Sci., vol.66, no.4, pp. 671-687, 2003.

[6] R. Baraniuk, M. Davenport, R. DeVore and M. Wakin, A simple proof of the restricted isometry property for random matrices, Constr. Approx., vol.28, no.3, pp. 253-263, 2008.

[7] M. Bregonzio, T. Xiang and S. Gong, Fusing appearance and distribution information of interest points for action recognition, Pattern Recogn., vol.45, no.3, pp. 1220-1234, 2012.. 